\title{
Resolución temporal visual en pacientes con anemia drepanocítica
}

\author{
Raúl Martínez Triana' \\ Universidad de La Habana, Cuba \\ Karen Reytor Alfonso \\ Universidad de La Habana, Cuba
}

Ailyn García Hernández

Universidad de La Habana, Cuba

Teresita Machado Almeida

Universidad de La Habana, Cuba

Diunaisy Rodríguez González

Universidad de La Habana, Cuba

Alain Risco Andrade

Universidad de La Habana, Cuba

\begin{abstract}
Resumen: El desarrollo del Sistema Nervioso (SN) está afectado en los pacientes con Anemia Drepanocítica (AD). La Resolución Temporal Visual (RTV) es considerada como una medida de la velocidad de procesamiento y de activación cortical. Se utiliza como indicador general del funcionamiento del SN y puede evaluarse mediante la Frecuencia Crítica de Fusión (FCF). Se midió la FCF en 84 pacientes sin antecedentes de eventos neurológicos, en edades entre 8-68 años, y en controles sanos. Se midió el valor de la hemoglobina $(\mathrm{Hb})$ de los pacientes al momento de la evaluación neuropsicológica. La FCF de los pacientes fue significativamente más baja. La edad correlacionó inversamente con la FCF; no se hallaron associaciones estatisticamente significativas entre los valores de la $\mathrm{Hb}$ y los resultados de la FCF. Se concluye que la RTV está disminuida en los pacientes con AD, lo que sugiere la existencia de afectación del neurodesarrollo como resultado de la enfermedad.
\end{abstract}

Palabras clave: anemia drepanocítica; neurodesarrollo; neurocognición; resolución temporal visual.

\section{VISUAL TEMPORAL RESOLUTION IN PATIENTS WITH SICKLE CELL ANEMIA}

\begin{abstract}
The Nervous System (NS) is affected in patients with Sickle Cell Anemia (SCA). The Visual Temporal Resolution (VTR) is considered as a measure of processing speed and cortical activation. It is used as general indicator of NS functioning, and it can be tested by Critical Frequency Fusion (CFF). The CFF was examined in 84 patients without previous neurologic events and the same amount in healthy subjects. The participants were ranging in age from 8 to 68 . The patients' hemoglobin level was measured at the moment of the neuropsychological assessment. The patients' CFF was significantly lower than healthy people. Age correlated inversely with CFF, and statistically
\end{abstract}

1 Endereço para correspondência: Raúl Martinez Triana. Instituto de Hematologia e Inmunologia. Apartado 8070, CP 10800. La Habana, Cuba. Telefone: (537) 643.8268. Fax (537) 644.2334. E-mail: raul.martinez@infomed. sld.cu 
significant associations between hemoglobin and CFF was not found. We concluded that VTR is diminished en patients with SCA, which suggests that neurodevelopment is affected due to SCA.

Keywords: sickle cell anemia; neurodevelopment; neurocognition; visual temporal resolution.

RESOLUÇÃO TEMPORAL VISUAL EM PACIENTES COM ANEMIA DREPANOCÍTICA

\begin{abstract}
Resumo: O desenvolvimento do Sistema Nervoso (SN) está afetado nos pacientes com Anemia Drepanocítica (AD). A Resolução Temporal Visual (RTV) é considerada uma medida da velocidade de processamento e de ativação cortical. Como indicador geral do funcionamento do SN essa medida é utilizada e pode ser mensurada mediante a Frequência Crítica de Fusão (FCF). Foi verificada a FCF em uma amostra de 84 pacientes sem antecedentes de eventos neurológicos, com idades entre 8 e 68 anos e em controles sadios. Mensurou-se o valor da hemoglobina $(\mathrm{Hb})$ dos pacientes durante a avaliação neuropsicológica. A FCF dos pacientes foi significativamente mais baixa. A idade correlacionou inversamente com a FCF; não foram encontradas associações estatisticamente significativas entre os valores da hemoglobina e os resultados da FCF. Conclui-se que a RTV está diminuída nos pacientes com AD sugerindo que há afetação do neurodesenvolvimento como resultado da doença.
\end{abstract}

Palavras-chave: reciprocidade; cooperação; retaliação; medida; validade.

\title{
Introducción
}

La drepanocitosis es un desorden genético caracterizado por defectos en la hemoglobina que dificultan la distribución de oxígeno y provocan eventos vaso-oclusivos. Estos eventos causan dolor y dañan múltiples órganos (Crosby, Quinn, \& Kalinyak, 2015). El SS (anemia drepanocítica) es el genotipo más común. Otras formas son: SC, $\mathrm{SB}^{0}$ y $\mathrm{SB}^{+}$. Los subtipos $\mathrm{SS}_{\text {y }} \mathrm{SB}^{0}$ están asociados con una elevada ocurrencia de problemas neurológicos, incluidos infartos cerebrales clínicos o silentes (Ballas et al., 2012) y pobre funcionamiento cognitivo desde la temprana infancia (Schatz \& Roberts, 2007). Los accidentes cerebrovasculares se presentan como una de las complicaciones más importantes de la drepanocitosis, que limita el desarrollo potencial pleno de niños y adultos (Detaum, Armstrong et al., 2012) La disfunción neurocognitiva está bien documentada en niños con drepanocitosis (Schatz, Finke, Kellett, \& Kramer, 2002) y aunque puede ser una de las consecuencias más importantes de esta enfermedad no ha sido muy estudiada en adultos (Vichinsky et al., 2010).

Los complejos procesos sistémicos de la hipoxia y la inflamación asociados también a la drepanocitosis son condiciones anormales que afectan el neurodesarrollo (Ianlampietro, Giovannetti, \& Tarazi, 2014). Existen evidencias que sugieren que en los pacientes con Anemia Drepanocítica (AD) la desaturación crónica de oxígeno en la hemoglobina, la enfermedad cerebrovascular y los frecuentes infartos, afectan el desarrollo del sistema nervioso desde etapas muy tempranas (Schatz, McClellan, Puffer, Johnson, \& Roberts, 2008).

Uno de los indicadores del funcionamiento del sistema nervioso es la velocidad de procesamiento. La calidad de la transmisión neural constituye una premisa básica para 
la adecuada interacción con el medio externo e interno. El sistema visual ha evolucionado como un mecanismo excelente en la adaptación a un medio ambiente cambiante y dinámico y la resolución con la cual un organismo sea capaz de captar y procesar la información visual va a determinar su habilidad para interactuar con los eventos que lo rodean (Healy, McNally, Ruxton, Cooper, \& Jackson, 2013). La Resolución Temporal Visual (RTV) se pone en evidencia en la capacidad de distinguir dos estímulos luminosos separados en el tiempo. La frecuencia en que una luz intermitente se percibe como continua es conocida como Frecuencia Critica de Fusión (FCF) (Seitz, Nanez, Holloway, \& Watanabe, 2005). La RTV es considerada como una medida de la velocidad de procesamiento del sistema nervioso y del nivel de activación cortical (Nardella, Rocchi, Conte, Bologna, Suppa, \& Berardelli, 2014) y se ha confirmado que la determinación de la FCF es una prueba confiable para la investigación de la RTV (Petukhov, Rozhentsov, \& Aliev, 2007).

En el presente artículo se utiliza la FCF para estudiar la RTV en pacientes con AD. Se hipotetiza que los pacientes puedan presentar una FCF mas baja que las personas sanas. No hemos encontrado evidencias de que se haya estudiado anteriormente la RTV en pacientes con drepanocitosis.

\section{Método}

\section{Participantes}

Tabla I. Características sociodemográficas de los pacientes estudiados.

\begin{tabular}{lccc} 
& $\begin{array}{c}\text { Adultos } \\
(>=18 \text { años })\end{array}$ & $\begin{array}{c}\text { Niños } \\
(>18 \text { años })\end{array}$ & Total \\
\hline Hombres & 20 & Sexo & 39 \\
\hline Mujeres & 35 & 19 & 45 \\
\hline Universitario & 20 & 10 & 13.1 \\
\hline Medio-superior & 61.8 & - & 42.9 \\
\hline Medio & 10.9 & 6.9 & 22.6 \\
\hline Primaria & 7.3 & 44.8 & 21.4
\end{tabular}

Fonte: Elaborada pelos autores.

Participaron 84 pacientes con AD en edades entre 8-68 años $(M=29.93, D E=16.97)$, que acudieron a consulta externa del IHI y que no tenían antecedentes de lesiones 
comprobadas del sistema nervioso. Otras características sociodemográficas de estos se muestran en la Tabla 1. Los resultados obtenidos por los pacientes fueron comparados con los de personas saludables que se parearon teniendo en cuenta; edad, sexo y nivel escolar. El grupo de sujetos sanos fue obtenido entre los trabajadores del Instituto de Hematología e Inmunología y alumnos y maestros de una escuela primaria cercana al mismo. El estudio fue aprobado por el Comité de Ética de la institución y se obtuvo el consentimiento informado de cada sujeto adulto y de los padres de los niños que participaron. Todos los participantes declararon que tenían una adecuada agudeza visual sin presentar significativos problemas oftalmológicos.

\section{Instrumento}

En un inicio la determinación de la FCF fue comprendida solo como un examen oftalmológico, usado para medir la agudeza visual y examinar las lesiones ópticas nerviosas. Esta prueba mide la frecuencia con que cada persona percibe que una luz fusionada/simple se convierte en una luz parpadeante (método descendente). Usualmente esta determinación se realiza múltiples veces (usualmente de 8-10) para permitir el cálculo de la media y la desviación estándar. Puede usarse también de modo inverso, donde la persona determina la frecuencia en que una luz parpadeante se convierte en continua o fusionada.

\section{Procedimiento}

Figura I. Medias grupales de las FCF obtenidas en cada una de las diez determinaciones.

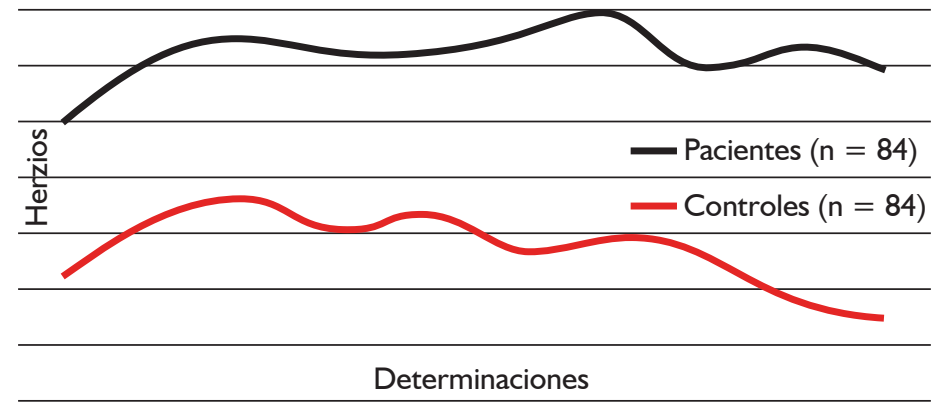

Fonte: Elaborada pelos autores.

Las aplicaciones se realizaron en un local poco iluminado, en condiciones confortables y de privacidad. Los pacientes fueron todos evaluados en la sesión de la tarde cuando asistieron a la consulta médica periódica en el Instituto de Hematología e 
Inmunología. El grupo de personas sanas fue evaluado en las mismas condiciones. Todos los participantes realizaron un entrenamiento de tres intentos descendentes previo a la realización de la prueba. Para la determinación del CFF de cada sujeto se utilizó la forma de visión binocular a $30 \mathrm{~cm}$ de distancia sobre un punto de color rojo de $2 \mathrm{~mm}$. Se determinó en cada sujeto la media de 10 determinaciones descendentes desde $60 \mathrm{~Hz}$ (rango posible de $60 \mathrm{~Hz}-10 \mathrm{~Hz}$ ). El equipo utilizado para medir el punto de fusión crítica fue un Flicker electrónico digital modelo x16 de la TakeiKiki, Co, LTD.

De los pacientes se obtuvo el nivel de $\mathrm{Hb}$ dentro de las 24 horas previas al momento de la determinación del FCF.

\section{Los resultados}

Tabla 2. Resultados de las comparaciones de medias realizadas.

\begin{tabular}{|c|c|c|c|c|c|c|c|}
\hline & \multirow[b]{2}{*}{$\mathrm{N}$} & \multicolumn{2}{|c|}{ Pacientes } & \multicolumn{2}{|c|}{ Controles } & \multicolumn{2}{|c|}{ Significación } \\
\hline & & Media & $\mathrm{DE}$ & Media & $\mathrm{DE}$ & $\mathrm{t}=$ & $\mathrm{p}=$ \\
\hline Grupo total & 84 & 27,608 & 5,433 & 31,177 & 3,781 & $-4,942$ & 0,000 \\
\hline$<18$ años & 29 & 31,209 & 5,191 & 33,468 & 2,159 & 2,164 & 0,035 \\
\hline$>=18$ años & 55 & 25,709 & 4,557 & 29,969 & 3,906 & $-5,264$ & 0.000 \\
\hline Varones & 39 & 28,455 & 5,591 & 31,990 & 3,027 & $-3,472$ & ,001 \\
\hline Hembras & 45 & 26,873 & 5,244 & 30,472 & 4,239 & $-3,580$ & , 001 \\
\hline
\end{tabular}

Fonte: Elaborada pelos autores.

Se comprobó que la FCF de los pacientes fue significativamente más baja que la de las personas sanas $(t=-4.942, p=0.000)$; resultados similares se mostraron al comparar las personas del mismo sexo entre ambos grupos (Tabla 2). La media obtenida, para cada una de las diez determinaciones que realizó a cada sujeto, también fueron significativamente inferiores en los pacientes ( $p<0.005)$ (Figura 1). La comparación de medias de los resultados entre niños y adultos mostró que estos últimos tienen una FCF significativamente más baja ( $t=-5.01 \mathrm{p}=0.000)$. Este efecto se muestra similar en el grupo de personas sanas $(t=-5.286, p=0.000$ ) aunque en los pacientes esta diferencia es mayor (Tabla 3). La variable sexo no mostró influencia en la comparación de medias entre pacientes, mientras que la edad correlacionó inversamente de forma significativa con la FCF media de estos $(r=-0.54 p=0.00)$. 
Tabla 3. Comparación de medias entre niños y adultos en pacientes y controles.

\begin{tabular}{llllllll} 
& Niños & & Adultos & & Significación & $\begin{array}{l}\text { Diferencia } \\
\text { entre las } \\
\text { medias }\end{array}$ \\
& Media & DE & Media & DE & $\mathrm{t}=$ & $\mathrm{p}=$ & \\
\hline Pacientes & 31,209 & 5,191 & 25,709 & 4,557 & $-5,010$ &, 000 & $-5,500$ \\
\hline Controles & 33,468 & 2,158 & 29,969 & 3,906 & $-5,286$ &, 000 & $-3,498$ \\
\hline
\end{tabular}

Fonte: Elaborada pelos autores.

Respecto al nivel de hemoglobina de los pacientes al momento de la evaluación, no se halló relación entre este y los resultados en la FCF $(r=0.048 ; p=0.663)$.

\section{Discusión}

Los resultados demuestran que la RTV está afectada en pacientes con AD. Se conoce que el neurodesarrollo bajo las condiciones fisiopatológicas de la AD puede ser la causa del daño específico neuronal en un órgano sensorial o general; lo que podría explicar que los pacientes del presente estudio muestren una RTV disminuida. En esta misma dirección Colombatti et al. (2015), en un trabajo reciente, mostraron que los potenciales evocados cognitivos y las redes neurales en los niños $A D$ no son normales, y sugirió que la respuesta cognitiva ante estímulos auditivos podía estar retrasada (Colombatti et al., 2015). El hecho de que muchas de las complicaciones de la drepanocitosis ocurren en momentos tempranos de la vida lleva a considerar el impacto que puede tener esta enfermedad sobre el desarrollo del sistema nervioso, sin embargo la gran mayoría de los estudios que abordan los efectos neurológicos de la misma no lo enfocan desde el neurodesarrollo.

El mapeo de la activación de la corteza cerebral en niños con $A D$, utilizando la secuencia BOLD (por sus siglas en inglés, Blood Oxygen Level Dependent), ha detectado cambios focales en la reducción del cortex primario visual en respuesta a la estimulación fótica, e incluso se ha determinado la existencia de relación entre esto y el resultado en pruebas cognitivas (Zou et al., 2011). El hecho de que ante una simple estimulación sensorial, las medidas de inteligencia global estuviesen relacionadas con el BOLD sugiere que la respuesta cortical visual pudiera llegar a considerarse un indicador de una respuesta globalmente afectada en los pacientes con AD.

Como resultado de las condiciones anormales durante el neurodesarrollo, la afectación en las vías aferentes puede ser causa de una respuesta disminuida del sistema nervioso en estos pacientes. Se ha observado en animales adultos de laboratorio la 
presencia de un daño permanente en la retina, debido a la hipoxia sufrida en estadios tempranos en el neurodesarrollo; estos animales mostraron una FCF reducida en comparación con los controles utilizados (Bourque, Kuny, Reyes, Davidge, \& Sauvé, 2013). En la AD las complicaciones oftalmológicas son relativamente comunes y pueden no ser detectadas en sus estadios tempranos (Ballas et al., 2012). En este trabajo solo controlamos esta variable a partir de la declaración de los propios sujetos. En próximos estudios debe usarse una valoración oftalmológica especializada para estimar su relación o no con la disminución de la RTV en estos pacientes. La condición de hipoxia tisular por anemia de los pacientes con drepanocitosis constituye otro de los elementos que caracterizan el medio en que ocurre el neurodesarrollo en ellos lo que se ha considerado que puede contribuir a la formación de redes neuronales alteradas desde la infancia (Colombatti et al., 2015).

Varios estudios han informado la relación existente entre el déficit de oxígeno y la FCF. Ha sido demostrada la relación entre la hipoxemia y la FCF en pacientes cardiópatas (Aisenberg, Rosenthal, Wolff, \& Nadas, 1974), y más recientemente Kot et al. (2015) concluyó que las personas que habían sido sometidas a condiciones de bajos niveles de $\mathrm{O}_{2}$, tenían un bajo rendimiento en las pruebas para determinar la FCF. En enfermos con drepanocitosis se ha relacionado el rendimiento neurocognitivo con la severidad de la anemia (Vichinsky et al., 2010) y se ha afirmado que la patofisiología de la AD puede interferir con la función cerebral y afectar el desarrollo intelectual aun en ausencia de infartos cerebrales a través de la hipoxia característica en esta enfermedad (Crosby et al., 2015). Sin embargo, en este trabajo no se halló relación entre los valores de $\mathrm{Hb}$ en el momento de la evaluación y las determinaciones FCF. Se hace necesario estudiar si la historia de los valores de $\mathrm{Hb}$, utilizados como un indicador de la evolución de la anemia en estos pacientes, muestra alguna relación con la FCF y no solo ceñirse a la medición de la $\mathrm{Hb}$ existente en el momento del estudio. La disminución de la FCF hallada en estos pacientes respecto a los controles sanos, pudiera estar más relacionada con problemas en el neurodesarrollo que con un déficit de oxígeno en el momento de la evaluación.

También se obtuvo que la diferencia entre el FCF de los niños y los adultos fue mayor en los pacientes que en los sujetos no enfermos estudiados. Es conocido que los niños pueden detectar cambios temporales en la estimulación visual con mayor agudeza que los adultos (Ellemberg, Lewis, Liu, \& Maurer, 1999) y ha sido establecido que en sujetos normales existe una disminución del FCF con la edad (Bernardi, Costa, \& Shiroma, 2007). Las redes neurales, incluyendo los sistemas sensoriales como la visión, pueden estar propensas a afectaciones durante el embarazo debido al hecho de que su desarrollo y maduración son altamente dependientes del contexto. Existen reportes que demuestran que un ambiente uterino no óptimo se asocia con alteraciones de la sensibilidad en la percepción del color, la agudeza y el contraste (Bourque et al., 2013). Algunos factores de riesgo para la disfunción cerebral isquémica en la anemia drepanocítica incrementan la probabilidad de daño neurocognitivo con la 
edad como son la hipoxia y la anemia crónica que han sido asociados, en la población general, con trastornos en la oxigenación cerebral y la perfusión (Kato, 2012). Se ha reportado que personas adultas con AD muestran un peor rendimiento en pruebas neurocognitivas que la población sana y se ha comprobado que la edad está asociada a la disminución de la ejecución cognitiva (Vichinsky et al., 2010). La velocidad de procesamiento, la memoria de trabajo, el $\mathrm{Cl}$ y funciones ejecutivas se encuentran entre las que muestran mayor deterioro al compararlas con controles en las personas adultas (Vichinsky et al., 2010). Los resultados del presente estudio sugieren que este deterioro puede expresarse en una mayor disminución de la RTV en estos pacientes que en las personas sanas.

Futuros trabajos sobre la FCF en pacientes con drepanocitosis deben precisar la posible relación de esta prueba con determinaciones del rendimiento neurocognitivo y exploración neurológica, con la intención de utilizar este test económico y de fácil aplicación como posible indicador de afectación neurocognitiva. Esto contribuiría al mejoramiento de la atención periódica e integral de las personas con esta enfermedad.

\section{Referências}

Aisenberg, R. B., Rosenthal, A., Wolff, P. H., \& Nadas, A. S. (1974). Hypoxemia and Critical Flicker Frequency in Congenital Heart Disease. Am J Dis Child., 128(3), 335-338.

Ballas, S., Kesen, M., Goldberg, M., Lutty, G., Dampier, C., Osunkwo, I. et al. (2012). Beyond the Definitions of the Phenotypic Complications of Sickle Cell Disease: An Update on Management. Scientific World Journal, 2012, 949535.

Bernardi, L., Costa, V. P., \& Shiroma, L. O. (2007). Flicker perimetry in healthy subjects: influence of age and gender, learning effect and short-term fluctuation. Arq Bras Oftalmol., 70, 91-99.

Bourque, S., Kuny, S., Reyes, L., Davidge, S., \& Sauvé, Y. (2013). Prenatal Hypoxia Is Associated with Long-Term Retinal Dysfunction in Rats. PLoS One, 8(4), 61861.

Colombatti, R., Ermani, M., Rampazzo, P., Manara, R., Montanaro, M., Basso, G. et al. (2015). Cognitive evoked potentials and neural networks are abnormal in children with sickle cell disease and not related to the degree of anaemia, pain and silent infarcts. British Journal of Haematology, 169, 597-600.

Crosby, L. E., Quinn, C. T., \& Kalinyak, K. A. (2015). A Biopsychosocial Model for the Management of Patients With Sickle-Cell Disease Transitioning to Adult Medical Care. Advances in Therapy, 32(4), 293-305. 
Detaum, M., Armstrong, F. D., McKinstry, R. C., Ware, R. E., Vichinsky, E., \& Kirkham, F. J. (2012). Silent cerebral infarcts: a review on a prevalent and progresive cause of neurologic injury in sickle cell disease. Blood, 119, 4587-4596.

Ellemberg D., Lewis, T. L., Liu, C. H., \& Maurer, D. (1999). Development of spatial and temporal vision during childhood. Vision Research, 39, 2325-2333.

Healy, K., McNally, L., Ruxton, G. D., Cooper, N., \& Jackson, A. L. (2013). Metabolic rate and body size are linked with perception of temporal information. Anim Behav., 86(4), 685-696.

Ianlampietro, M., Giovannetti, T., \& Tarazi, R. (2014). Hypoxia and inflammation in children with sickle cell disease: implications for hippocampal functioning and episodic memory. Neuropsychol Rev, 24(2), 252-265.

Kato, G. J. (2012). Anemia, age, desaturation and impaired neurocognition in Sickle Cell Anemia. Pediatr Blood Cancer, 59(5), 773-774.

Kot, J., Winklewski, P., Sicko, Z., \& Tkachenko, Y. (2015). Effect of oxygen on neuronal excitability measured by critical flicker fusion frequency is dose dependent. Journal of Clinical and Experimental Neuropsychology, 37(3), 276-284.

Nardella, A., Rocchi, L., Conte, A., Bologna, M., Suppa, A., \& Berardelli, A. (2014). Inferior Parietal Lobule Encodes Visual Temporal Resolution Processes Contributing to the Critical Flicker Frequency Threshold in Humans. PLoS ONE, 9(6), e98948.

Petukhov, I. V., Rozhentsov, V. V., \& Aliev, M. T. (2007). On the accuracy of evaluations of temporal characteristics of visual perception. Bull Exp Biol Med., 144, 267-268.

Schatz, J., Finke, R. L., Kellett, J. M., \& Kramer, J. H. (2002). Cognitive functioning in children with sickle cell disease: a meta-analysis. J Pediatr Psychol, 27, 739-748.

Schatz, J. \& Roberts, C. W. (2007). Neurobehavioral impact of sickle cell disease in early chilhood. J Int Neuropsychol Soc., 13, 933-943.

Schatz, J., McClellan, C. B., Puffer, E. S., Johnson, K., \& Roberts, C.W. (2008). Neurodevelopmental screening in toddlers and early preschoolers with sickle cell disease. J Child Neurol., 23, 44-50.

Seitz, A. R., Nanez, J. E., Holloway, S. R., \& Watanabe, T. (2005). Visual experience can substantially alter critical flicker fusion thresholds. Hum Psychopharmaco, 20, 55-60.

Vichinsky, E. P., Neumayr, L. D., Gold, J. I., Weiner, M. W., Rule, R. R., Truran, D. et al. (2010). Neuropsychological dysfunction and neuroimaging abnormalities in neurologically intact adults with sickle cell anemia. JAMA, 303, 1823-1831. 
Zou, P., Helton, K. J., Smeltzer, M., Li, C. S., Conklin, H. M., Gajjar, A. et al. (2011) Hemodynamic responses to visual stimulation in children with Sickle Cell Anemia. Brain Imaging Behavior, 5(4), 295-306.

Sumisión: 8.5.2017

Aceptación: 20.6.2017 